\title{
Effect of scintillations in curvature sensing
}

\author{
V. V. Voitsekhovich and L. J. Sánchez
}

\author{
Instituto de Astronomía - UNAM, Apdo. Postal 70-264, Cd. Universitaria, 04510 México D.F., México \\ e-mail: leonardo@astroscu.unam.mx
}

Received 13 August 2002 / Accepted 7 November 2002

\begin{abstract}
The effect of scintillations on the curvature sensing which is used in astronomic adaptive systems is investigated. It is shown that the magnitude of the effect depends on two atmospheric turbulence parameters: on the Fried parameter which characterizes the seeing conditions, and on the turbulence inner scale which is responsible for small-sized turbulence inhomogeneities. Depending on the turbulence conditions, the magnitude of the effect ranges from $3 \%$ to $20 \%$, while for the typical ones it is of the order of $10 \%-15 \%$.
\end{abstract}

Key words. atmospheric effects - instrumentation: adaptive optics - instrumentation: high angular resolution

\section{Introduction}

The curvature sensing is a promising technique for wavefront measurements suggested by F. Roddier (Roddier 1987). This method is getting more and more popular in astronomic adaptive optics because it combines a relatively simple experimental setup with good accuracy of measurements. The theoretical background providing the relationship between the sensor signal and the wavefront to be reconstructed was developed for the case of uniform illumination assuming at the very beginning that the amplitude fluctuations of incoming fields produce a negligible effect on the quality of wavefront reconstruction. While for laboratory measurements this assumption is valid, in real astronomical applications, however, the curvature signal is always affected by atmospherically-induced scintillations and it is important to know how strong this effect is.

In order to calculate the magnitude of the effect, we derive first the generalized curvature sensing equation which is valid for the case of non-uniform illumination. As it will be seen through the paper, compared to the original one, this generalized equation has just one additional term which is responsible for the effect of scintillations. Calculating a contribution of this term to the curvature signal we get a general expression for the magnitude of the effect of interest. Then making use of this general expression we estimate quantitatively the magnitude of the effect for the weak-turbulence conditions that is the typical case for astronomical observations.

\section{Curvature sensing equation for the case of non-uniform illumination}

The curvature sensing method was suggested by F. Roddier (Roddier 1987) and its optical setup is shown in Fig. 1.

Send offprint requests to: V. V. Voitsekhovich, e-mail: voisteko@astroscu.unam.mx

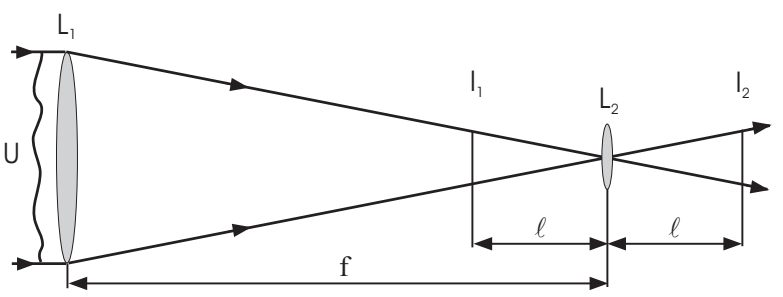

Fig. 1. Curvature sensing experimental setup. The distorted input wavefield passes the lens $L_{1}$ with focal length $f$. The sensor signal is composed from the intensity distributions $I_{1}$ and $I_{2}$ of two symmetrically-defocused images. The focal length of the lens $L_{2}$ is equal to $f / 2$.

This method allows one to reconstruct the phase $S$ of the input field $U$ using the intensity measurements $I_{1}$ and $I_{2}$ of two symmetrically-defocused images. The curvature sensing equation providing a relationship between the sensor signal $\eta$ and the phase $S$ to be reconstructed is

$\eta(\boldsymbol{r})=\frac{I_{2}(\boldsymbol{r})-I_{1}(-\boldsymbol{r})}{I_{2}(\boldsymbol{r})+I_{1}(-\boldsymbol{r})}=-\frac{f(f-l)}{k l}\left[\Delta S(\boldsymbol{\rho})+\frac{\partial S(\boldsymbol{\rho})}{\partial \boldsymbol{n}} \delta_{\mathrm{c}}\right]$

where $k$ is the wavenumber, $\delta_{\mathrm{c}}$ denotes a linear impulse distribution around the pupil edge, and the right-side functions are evaluated for $\boldsymbol{\rho}=f \boldsymbol{r} / l$.

Equation (1) was derived in the geometric optics approximation for the case of uniform pupil illumination. However in order to calculate the effect of scintillations on the sensor signal we need to derive a more general equation which will be valid for the case of non-uniform illumination.

Following Roddier's derivations (Roddier 1987) we can write the intensities $I_{1}$ and $I_{2}$ as

$\begin{aligned} I_{1}(\boldsymbol{r})= & -\left(\frac{\alpha}{2 \pi}\right)^{2} \int \mathrm{d}^{2} \varkappa \exp \left\{i\left(\alpha \boldsymbol{r} \boldsymbol{\varkappa}-\frac{\beta}{2} \varkappa^{2}\right)\right\} \\ & \times \int \mathrm{d}^{2} \rho P(\boldsymbol{\rho}) P(\boldsymbol{\rho}+\boldsymbol{\varkappa}) U(\boldsymbol{\rho}) U^{*}(\boldsymbol{\rho}+\boldsymbol{\varkappa}) \exp \{-i \beta \boldsymbol{\rho} \boldsymbol{\varkappa}\}\end{aligned}$ 


$$
\begin{aligned}
& I_{2}(\boldsymbol{r})=-\left(\frac{\alpha}{2 \pi}\right)^{2} \int \mathrm{d}^{2} \varkappa \exp \left\{i\left(\alpha \boldsymbol{r} \boldsymbol{\varkappa}+\frac{\beta}{2} \varkappa^{2}\right)\right\} \\
& \times \int \mathrm{d}^{2} \rho P(\boldsymbol{\rho}) P(\boldsymbol{\rho}+\varkappa) U(\boldsymbol{\rho}) U^{*}(\boldsymbol{\rho}+\boldsymbol{\varkappa}) \exp \{i \beta \rho \varkappa\} \\
& \alpha=\frac{k}{f-l}, \beta=\frac{k l}{f(f-l)}
\end{aligned}
$$

where $U$ is the field at the pupil, and $P$ denotes the pupil function.

Roddier has shown (Roddier 1987) that Eqs. (2) and (3) can be significantly simplified applying their geometric optics approximation which is valid when

$l \gg \frac{2 \pi f(f-l)}{k r_{0}^{2}}$,

where $r_{0}$ is the correlation length of the input field.

If the condition (5) holds, the following approximations can be made

$U(\rho) U^{*}(\rho+\varkappa) \approx A(\rho)[A(\rho)+\varkappa \cdot \nabla A(\rho)][1-i \varkappa \cdot \nabla S(\rho)]$

$P(\rho) P(\rho+\varkappa) \approx P(\rho)$

$\exp \left(i \frac{\beta}{2} \varkappa^{2}\right) \approx 1$

where $A$ is the amplitude of the input field.

Substituting the approximated quantities (6) to Eqs. (2) and (3) and evaluating the integrals we get

$I_{1}(-\boldsymbol{r})=\frac{4 \pi^{2}}{\beta^{2}}\left\{A^{2}(\boldsymbol{\rho})+\frac{1}{\beta} \boldsymbol{\nabla}\left[P(\boldsymbol{\rho}) A^{2}(\boldsymbol{\rho}) \boldsymbol{\nabla} S(\boldsymbol{\rho})\right]\right\}$

$I_{2}(\boldsymbol{r})=\frac{4 \pi^{2}}{\beta^{2}}\left\{A^{2}(\boldsymbol{\rho})-\frac{1}{\beta} \boldsymbol{\nabla}\left[P(\boldsymbol{\rho}) A^{2}(\boldsymbol{\rho}) \boldsymbol{\nabla} S(\boldsymbol{\rho})\right]\right\}$

where $\boldsymbol{\rho}=f \boldsymbol{r} / l$.

Calculating the sensor signal $\eta$, we get from Eqs. (7) and (8)

$$
\begin{aligned}
\eta(\boldsymbol{r}) & =\frac{I_{2}(\boldsymbol{r})-I_{1}(-\boldsymbol{r})}{I_{2}(\boldsymbol{r})+I_{1}(-\boldsymbol{r})} \\
& =-\frac{f(f-l)}{k l}\left[\triangle S(\boldsymbol{\rho})+\frac{\partial S(\boldsymbol{\rho})}{\partial \boldsymbol{n}} \delta_{\mathrm{c}}+2 \nabla \chi(\boldsymbol{\rho}) \nabla S(\boldsymbol{\rho})\right] \\
\chi(\boldsymbol{\rho}) & =\ln \left[A(\boldsymbol{\rho}) / A_{0}\right]
\end{aligned}
$$

where $\chi$ denotes the log-amplitude, and $\boldsymbol{\rho}=f \boldsymbol{r} / l$.

Equation (9) is a generalized curvature sensing equation for the case of non-uniform illumination. Comparing to the original Roddier's equation, it has just one additional term $2 \nabla \chi(\rho) \nabla S(\rho)$.

\section{Scintillation-induced error: Analytical derivations}

In order to define properly the effect of scintillations on the sensor signal, let us analyze first the structure of Eq. (9). As it follows from Eq. (9), the sensor signal is affected by three terms: the first one is the phase Laplacian $\Delta S$ (the curvature part of the sensor signal), the second one $\delta_{\mathrm{c}} \partial S / \partial \boldsymbol{n}$ describes the boundary signal, and the third one $2 \nabla \chi \nabla S$ is responsible for the scintillation effect (the scintillation term). Analyzing the equation structure one can see that its right-side part consists of two smooth terms $(\triangle S$ and $\nabla \chi \nabla S)$ which are defined over all the aperture and a singular one $\left(\delta_{\mathrm{c}} \partial S / \partial \boldsymbol{n}\right)$ which predicts the boundary signal as the delta-function located at the edge. In reality the boundary signal magnitude is finite and it is located inside a thin but a finite edge zone while the singularity appears because Eq. (9) has been derived in the geometric optics approximation. However such a singularity does not allow us to define properly the influence of scintillations on the boundary signal, so we restrict our attention to the effect of scintillations on the curvature part of the sensor signal. Also we calculate below the magnitude of the effect for weak-turbulence conditions only because it is the typical case for astronomical observations.

Everywhere, excluding the boundary, the sensor signal is affected by two terms: by the curvature term $\Delta S$ and by the scintillation term $2 \nabla \chi \nabla S$. Because the terms are additive, the scintillation effect on the curvature signal can be characterized as a ratio of the contributions of the corresponding terms to the sensor signal (in a statistical sense). Taking into account that both terms are the statistical quantities and that the turbulence-induced fluctuations are homogeneous, we define quantitatively the scintillation influence on the sensor signal as

$\sigma=\sqrt{\frac{\left\langle(2 \nabla \chi \nabla S)^{2}\right\rangle}{\left\langle(\triangle S)^{2}\right\rangle}}$

where $\langle\cdots\rangle$ stands for the statistical average.

In what follows we refer to the quantity $\sigma$ as a scintillation error.

Under the weak-turbulence conditions the quantities $\nabla \chi$ and $\nabla S$ are Gaussian, so we can write (Goodman 1985)

$\left\langle(\boldsymbol{\nabla} \chi \nabla S)^{2}\right\rangle=\langle\nabla S \nabla S\rangle\langle\nabla \chi \nabla \chi\rangle+2\langle\nabla \chi \nabla S\rangle^{2}$.

The quantities at the right-side part of Eq. (11) and $\left\langle(\Delta S)^{2}\right\rangle$ can be expressed through the corresponding correlation functions as

$\langle\nabla S \nabla S\rangle=\nabla_{\rho_{1}} \nabla_{\rho_{2}} B_{S}\left(\rho_{1}, \rho_{2}\right)_{\mid \rho_{1}=\rho_{2}}$,
$\langle\nabla \chi \nabla \chi\rangle=\nabla_{\rho_{1}} \nabla_{\rho_{2}} B_{\chi}\left(\rho_{1}, \rho_{2}\right)_{\mid \rho_{1}=\rho_{2}}$,
$\langle\nabla \chi \nabla S\rangle=\nabla_{\rho_{1}} \nabla_{\rho_{2}} B_{\chi S}\left(\rho_{1}, \rho_{2}\right)_{\mid \rho_{1}=\rho_{2}}$,
$\left\langle(\triangle S)^{2}\right\rangle=\triangle_{\rho_{1}} \triangle_{\rho_{2}} B_{S}\left(\boldsymbol{\rho}_{1}, \rho_{2}\right)_{\mid \rho_{1}=\rho_{2}}$,

where $B_{S}, B_{\chi}$, and $B_{\chi S}$ are the phase, log-amplitude, and logamplitude-phase correlation functions, respectively.

For the case of weak-turbulence conditions the above correlation functions can be written as (Tatarski 1968)

$B_{S}(\boldsymbol{\rho})=\pi k^{2} \int_{0}^{L} \mathrm{~d} z \int \mathrm{d}^{2} \varkappa \Phi_{n}(z, \varkappa) \exp (i \varkappa \rho)\left[1+\cos \left(\frac{z}{k} \varkappa^{2}\right)\right]$,

$B_{\chi}(\rho)=\pi k^{2} \int_{0}^{L} \mathrm{~d} z \int \mathrm{d}^{2} \varkappa \Phi_{n}(z, \varkappa) \exp (i \varkappa \rho)\left[1-\cos \left(\frac{z}{k} \varkappa^{2}\right)\right]$,

$B_{\chi S}(\rho)=\pi k^{2} \int_{0}^{L} \mathrm{~d} z \int \mathrm{d}^{2} \varkappa \Phi_{n}(z, \varkappa) \exp (i \varkappa \rho) \sin \left(\frac{z}{k} \varkappa^{2}\right)$,

$\rho=\rho_{1}-\rho_{2}$,

where $L$ is the propagation path length, and $\Phi_{n}$ denotes the spectrum of refractive-index fluctuations. 
At this point we need to make a proper choice of the spectrum $\Phi_{n}$. Usually the pure Kolmogorov spectrum $\Phi_{n} \sim \varkappa^{-11 / 3}$ is used for the turbulence-related calculations. Strictly speaking this spectrum is applicable only inside the inertial range of the turbulence (Tatarski 1968), but for many cases of practical concern when the calculated quantities are affected slightly by small and big-sized turbulence inhomogeneities, it gives correct results. However our case is a special one because the second phase derivatives are involved in the calculations. It is clear from the physical viewpoint that the higher the derivative order is, the more strongly its magnitude is affected by small-sized turbulence inhomogeneities. In other words, the contribution of high spatial frequencies is growing up with the increasing of the derivative order. However because the pure Kolmogorov spectrum allows for the existence of infinitively small turbulence inhomogeneities, it is not suitable for our calculations (for example, the variance of the second phase derivative calculated with the pure Kolmogorov spectrum is infinitively large). So we need to use such a modification of the Kolmogorov spectrum that restricts the minimum size of turbulence inhomogeneities. In what follows we use the classical Tatarski model (Tatarski 1968) which is expressed as

$\Phi_{n}(z, \varkappa)=0.033 C_{n}^{2}(z) \varkappa^{-11 / 3} \exp \left(-\varkappa^{2} / \varkappa_{m}^{2}\right)$, $\varkappa_{m}=5.92 / l_{0}$,

where $C_{n}^{2}(z)$ is the profile of the refractive-index structure characteristic, and $l_{0}$ is the inner scale of the turbulence.

Substituting (14) into (13), evaluating the corresponding integrals and taking into account (12), we get the following final results for the quantities of interest

$$
\begin{aligned}
&\langle\nabla S \nabla S\rangle= K_{0} \int_{0}^{L} \mathrm{~d} z C_{n}^{2}(z)\left(1+\mu^{2}\right)^{-1 / 12} \sin \left[\frac{1}{6} \arctan (\mu)\right], \\
&\langle\nabla \chi \nabla \chi\rangle= K_{0} \int_{0}^{L} \mathrm{~d} z C_{n}^{2}(z)\left\{1-\left(1+\mu^{2}\right)^{-1 / 12}\right. \\
&\left.\times \cos \left[\frac{1}{6} \arctan (\mu)\right]\right\}, \\
&\langle\nabla \chi \nabla S\rangle= K_{0} \int_{0}^{L} \mathrm{~d} z C_{n}^{2}(z)\left\{1+\left(1+\mu^{2}\right)^{-1 / 12}\right. \\
&\left.\times \cos \left[\frac{1}{6} \arctan (\mu)\right]\right\}, \\
&\left\langle(\Delta S)^{2}\right\rangle= K_{1} \int_{0}^{L} \mathrm{~d} z C_{n}^{2}(z)\left\{1+\left(1+\mu^{2}\right)^{-7 / 12}\right. \\
&\left.\times \cos \left[\frac{7}{6} \arctan (\mu)\right]\right\} \\
& K_{0}=0.033 \pi^{2} k^{2} \Gamma\left(\frac{1}{6}\right) \varkappa_{m}^{1 / 3}, \\
& K_{1}=0.033 \pi^{2} k^{2} \Gamma\left(\frac{7}{6}\right) \varkappa_{m}^{7 / 3}, \mu=\frac{z \varkappa_{m}^{2}}{k}
\end{aligned}
$$

where $\Gamma$ denotes the gamma-function.

\section{Calculation results}

As one can see from (15), we need to use a $C_{n}^{2}$ profile (turbulence strength profile) to be able to estimate numerically the

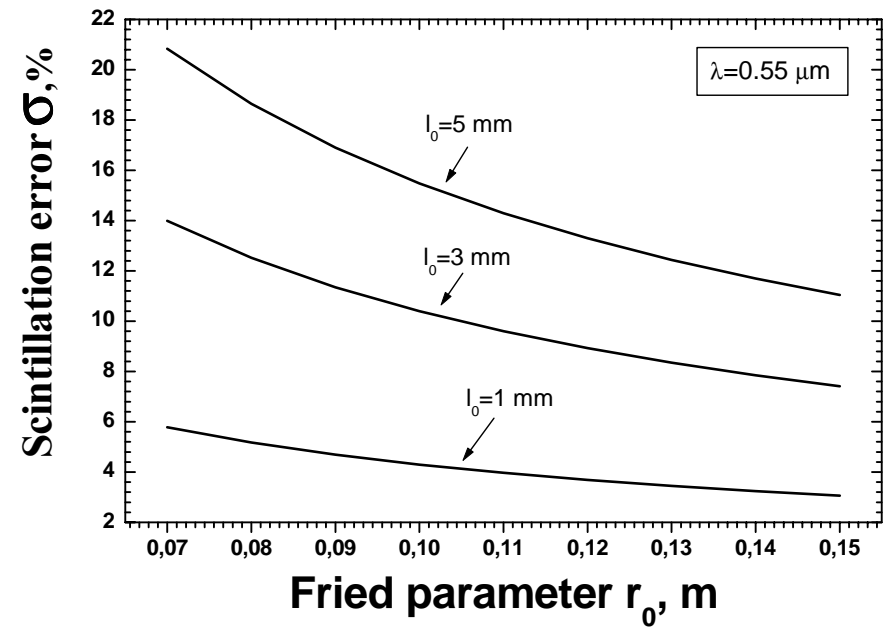

Fig. 2. Scintillation error $\sigma$ versus Fried parameter $r_{0}$ (wavelength $\lambda=$ $0.55 \mu \mathrm{m})$.

magnitude of the effect of interest. An appropriate analytical $C_{n}^{2}$ profile model derived on the base of experimental data is the Hufnagel model (Hufnagel 1974) which can be written as

$C_{n}^{2}(z)=C_{0} r_{0}^{-5 / 3} k^{-2}\left[\left(\frac{z}{z_{0}}\right)^{10} \exp \left\{-\frac{z}{z_{1}}\right\}+\exp \left\{-\frac{z}{z_{2}}\right\}\right]$

where $r_{0}$ is the Fried parameter, $k$ is the wavenumber, $C_{0}=$ $1.027 \times 10^{-3} \mathrm{~m}^{-1}, z_{0}=4.632 \times 10^{3} \mathrm{~m}, z_{1}=10^{3} \mathrm{~m}, z_{2}=$ $1.5 \times 10^{3} \mathrm{~m}$.

The calculated results of the scintillation error $\sigma$ given by (10) for the case of Hufnagel profile (16) are presented in Figs. 2 and 3.

Figure 2 presents the dependance of the scintillation error $\sigma$ on the Fried parameter $r_{0}$ for three values of the turbulence inner scale $l_{0}$ (see Eq. (14) for $l_{0}$ definition). The changing range of the Fried parameter $r_{0}$ is chosen in such a way that allows us to include observation conditions ranging from the poor seeing $r_{0}=0.07 \mathrm{~m}$ to the excellent seeing $r_{0}=0.15 \mathrm{~m}$ (the relationship between the Fried parameter and the image quality is given in Table 1 of Voitsekhovich et al. (2001). As one can see from Fig. 2, the magnitude of the scintillation error decreases when the seeing improves. It happens because when the seeing improves, the phase-log-amplitude correlation which is responsible for the contribution of the scintillation term $\nabla \chi \nabla S$ to the sensor signal decreases faster than the phase Laplacian variance.

Comparing the three graphs in Fig. 2, one can notice that the scintillation error is growing with increasing turbulence inner scale $l_{0}$. This effect is shown in more detail in Fig. 3 where the magnitude of the scintillation error versus the inner scale value is plotted. The physical reason for this effect is as follows. The contribution of the scintillation term $\nabla \chi \nabla S$ to the sensor signal is determined by the first derivatives, while the contribution of the Laplacian term $\Delta S$ depends on the second derivatives. As it has been mentioned in the previous section, the higher the derivative order, the more strongly its magnitude is affected by small-sized turbulence inhomogeneities, so the Laplacian term $\Delta S$ is affected more strongly by small-sized 


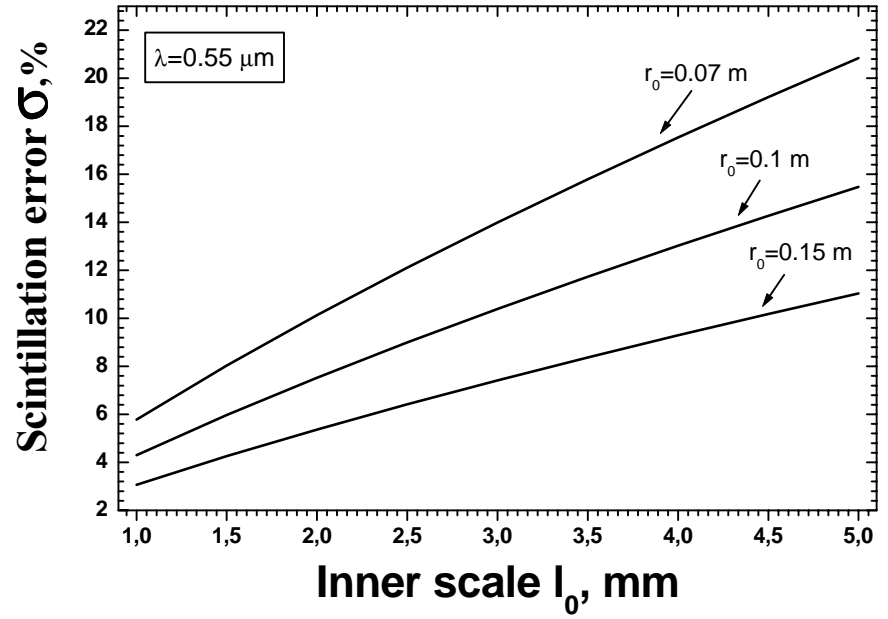

Fig. 3. Scintillation error $\sigma$ versus inner scale $l_{0}$ (wavelength $\lambda=$ $0.55 \mu \mathrm{m})$.

inhomogeneities than the scintillation term $\nabla \chi \nabla S$. When the inner scale value is increasing, the number of small-sized inhomogeneities is decreased. However because the Laplacian term depends more strongly on the number of small-sized inhomogeneities than the scintillation one, its magnitude is decreased faster. As a result, the scintillation error is increased with the increasing inner scale.

The Hufnagel $C_{n}^{2}$ model predicts a generalized behavior of the turbulence strength with the altitude that allows us to investigate the salient features of the effect of interest. However because the Hufnagel $C_{n}^{2}$ model is a smooth analytical approximation of averaged data, it does not take into account the pronounced layered structure observed in real profiles. Also the shapes of real $C_{n}^{2}$ profiles can differ from the Hufnagel one. In order to see how the above factors can affect the final results, we perform the calculations with the two experimental profiles plotted in Fig. 4. Both profiles were measured with the balloon technique at the same observatory (Haute Provence observatory, France) but at different times (the left profile was obtained at 06.06.1996, while the right one was obtained at 29.09.1997). Both profiles demonstrate the same integral turbulence strength (expressed through the Fried parameter $r_{0} \simeq 0.12 \mathrm{~m}$, and their shapes are also similar. However the turbulence observed at low altitude 29.09.1997 (Fig. 4, right panel) is stronger than that 06.06.1996 (Fig. 4, left panel). Although this difference seems not very big on the graph, it affects quite strongly the associated scintillation errors which are plotted in Fig. 5. From the physical viewpoint, the difference between the two plots in Fig. 5 can be explained as follows. The magnitude of the scintillation error is determined by the relative contributions of the Laplacian term $\triangle S$ and of the scintillation term $\nabla \chi \nabla S$ to the curvature signal (the bigger the Laplacian term relative to the scintillation one, the smaller the scintillation error is, and vis versa). However the main contribution to the magnitude of the scintillation term comes from high-altitude turbulence layers, while the Laplacian term is mainly affected by more lowlevel turbulence. So, since the profile at 29.09.1997 has more

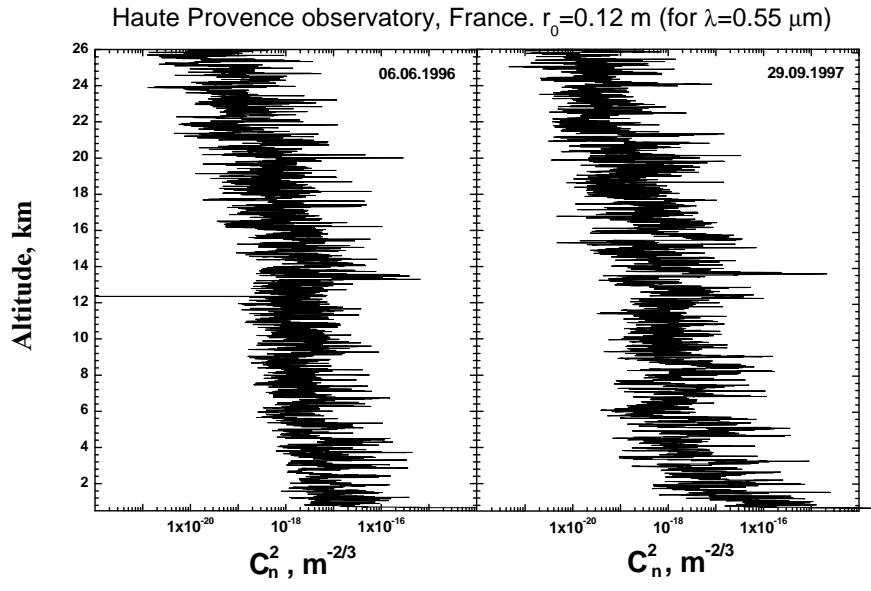

Fig. 4. Experimental $C_{n}^{2}$ profiles measured with the balloon technique at the Haute Provence Observatory, France.

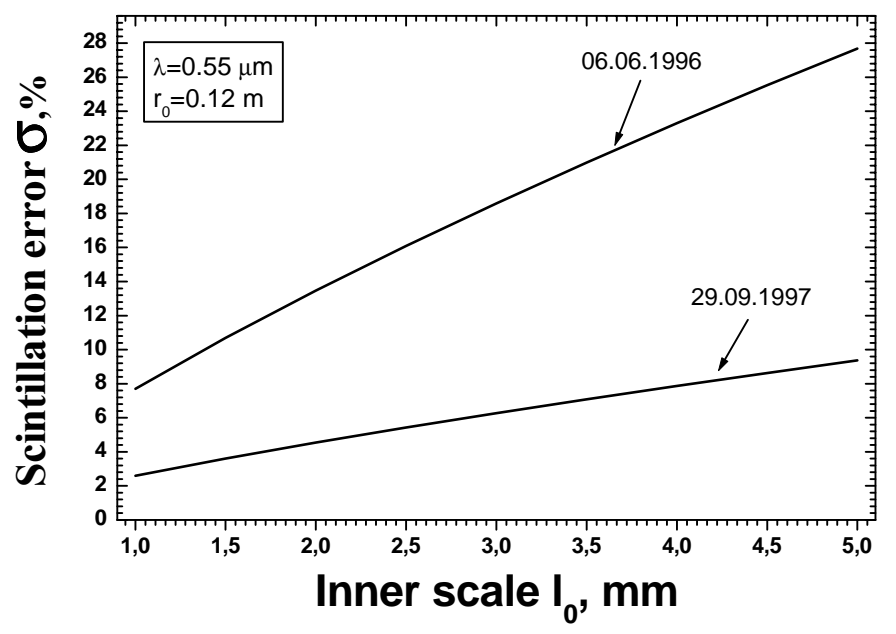

Fig. 5. Scintillation error $\sigma$ for the two experimentallymeasured $C_{n}^{2}$ profiles plotted in Fig. 4.

turbulence energy at low altitude level, the associated scintillation error is smaller.

\section{Conclusions}

The effect of scintillations on curvature sensing has been investigated. The analysis has been performed on the basis of a generalized curvature-sensing equation which is valid for the case of non-uniform illumination. Numerical calculations have been done for weak-turbulence conditions because it is the typical case for astronomical observations.

The calculations have been performed with both the Hufnagel $C_{n}^{2}$ profile model and with the two experimentally measured profiles as well. The calculated results with the Hufnagel $C_{n}^{2}$ profile have shown that the magnitude of the effect depends on two turbulence parameters: on the Fried 
parameter $r_{0}$ which characterizes the seeing conditions, and on the inner scale of the turbulence $l_{0}$ which is responsible for small-sized turbulence inhomogeneities. It has been shown that the magnitude of the effect is decreased when the seeing improves or when the inner scale decreases. Depending on the turbulence conditions, the magnitude of the effect ranges from $3 \%$ to $20 \%$, while for the typical ones $\left(r_{0} \simeq 0.13 \mathrm{~m}, l_{0} \simeq 3 \mathrm{~mm}\right)$ it is in order of $10 \%-15 \%$.

The calculated results with the experimentally measured $C_{n}^{2}$ profiles allow one to conclude that the magnitude of the effect depends on the turbulence strength distribution among the turbulence layers. Also these results allow one to arrive at a more general but preliminary conclusion: the bigger the amount of the turbulence strength concentrated at low altitude levels, the smaller the scintillation error is.
Acknowledgements. We are indebted to Dr. J. Vernin and Dr. M. Azouit for providing $C_{n}^{2}$ balloon data. This work received support from Consejo Nacional de Ciencia y Tecnología, (México), grant 34517-E.

\section{References}

Goodman, J. W. 1985, Statistical Optics (Wiley\&Sons, Inc.)

Hufnagel, R. E. 1974, in Optical Propagation through Turbulence (OSA, Washington, D.C., WA1)

Roddier, F. 1987, NOAO R\&D Note 87-3

Tatarski, V. I. 1968, The Effects of the Turbulent Atmosphere on Wave Propagation, National Science Foundation Report TT-68-50464

Voitsekhovich, V. V., Orlov, V. G., \& Sánchez, L. J. 2001, A\&A, 368, 1133 